\title{
Microscopy Core Facilities: Results of an International Survey
}

\section{Horst Wallrabe, ${ }^{1}$ Ammasi Periasamy, ${ }^{1,2}$ and Masilamani Elangovan ${ }^{3 *}$}

${ }^{1}$ The W.M. Keck Center for Cellular Imaging, Departments of Biology and ${ }^{2}$ Biomedical Engineering, University of Virginia, Charlottesville, VA 22904

${ }^{3}$ Idea Elan, 12801 Worldgate Dr., Suite 500, Herndon, VA 20170

*elango@ideaelan.com

\section{Introduction}

Core centers in the life sciences at academic institutions are well established [1], providing services for histology, sequencing, genomics, biostatistics, etc. Creating and running a microscopy core facility, however, presents unique challenges and opportunities $[2,3]$. Cutting edge, sophisticated instrumentation provides researchers with many opportunities to advance their research goals, while perhaps requiring expert advice, training, or consultation from core center staff. The requirements of instrument maintenance, data storage, analysis, interpretation of results, and facility finances put a premium on the technical and management skills of core center directors and staff. The heterogeneous nature of microscopy cores, most likely based on historical developments, leads to multiple units in some academic institutions and sparks calls for consolidation, streamlining of systems for greater efficiency, and better resource utilization [3].

This article presents results of a survey constructed to analyze the functionality and management of biological microscopy core facilities. The range of questions included in the survey covered various aspects of facility structure and organization, instrumentation, financial support, productivity tools, and user profiles. Analysis of these data provides insights into how best to organize and manage a microscopy core facility.

These survey data should provide institutions with some guidelines for operation of a core facility of this nature. Institutions newly adopting a shared resource system would benefit from input on how core facilities are run in different parts of the world. The responses allow new facilities to better understand the demands of a business-like environment combined with a technological service function when planning a new microscopy core center. Existing core facilities may find some interesting practices, which may help them to better manage their own organization, increase their numbers of users, work more efficiently, and possibly find new sources of financial support.

\section{Overview of Responding Microscopy Core Centers}

We solicited information from several hundred academic microscopy centers and received 175 responses from 22 countries. A total of 27 questions were posed. Data collection was facilitated by an online survey tool (www.surveymonky. com). The responses were separated into geographical regions, blinded, and then analyzed by the first author of this article. Although 175 centers participated in the survey, not all questions were answered either because they were not applicable or because the data were not available. In some cases, there was no explanation given for the lack of answers. Each table states the number of responders to a particular question, and this may be compared with the numbers in Table 1 . The cohorts answering may be different from one question to another. For some questions multiple choices were applicable so the totals may exceed $100 \%$.

Table 1 provides a summary profile of the responders. Of the 175 responders, $63 \%$ originated from North America (US-East $\sim 27 \%$, US-Central $\sim 19 \%$, US-West $\sim 11 \%$, Canada $\sim 6 \%$ ), $29 \%$ from Europe, and $8 \%$ from the Asia Pacific region (Asia, Australia, New Zealand). Table 1 breaks down responders by region in terms of percent of responders reporting the number of microscopy cores per institution, type and number of instruments, and whether the cores conduct their own research. Overall, some $40 \%$ have just one core with some differences between regions. About $10 \%$ have no core (data not shown) but have instruments spread across the institution. Not surprisingly, most cores have multiple systems; only about $10 \%$ report having $1-2$ systems. Nearly $90 \%$ report confocal systems. Responders describing themselves as primarily multiphoton facilities also each had confocals (data not shown). Moving to electron microscopy, $30 \%$ engage in scanning electron microscopy (SEM) and about 34\% use transmission electron microscopy (TEM). Forty-seven centers (27\%) combine light microscopy and electron microscopy even though these two techniques serve different purposes and require different specific skill sets, for example, need for EM specimen preparation. Almost half (48\%) of the core centers conduct their own research in many different areas, whereas the others do not get involved in research, acting primarily as service facilities. Those that are involved in research, report a wide spectrum of research activities: instrument design, instrument- and analysis-related software development, specific life-science projects (calcium imaging, yeast cells, mammalian cells, and plant cells), materials science, forensic projects, etc. Other than those institutions claiming not to have a core, some responders mentioned additional higher-end systems outside the cores that are attached to individual labs.

\section{Financial Aspects of Running a Core Facility}

The financial aspects of this survey covered operational expenses (including staff costs) and their funding, capital expenses of instrumentation and their funding, user fees, and what to do with old instruments. Managing this area is one of the most challenging tasks of the core director. The sources of funding reported in this survey display a varied pattern of government, institutional, foundation, and user/client contributions. 


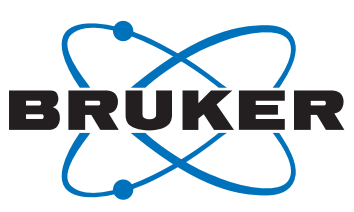

\section{5-in-1 fantasy: Giralope.}

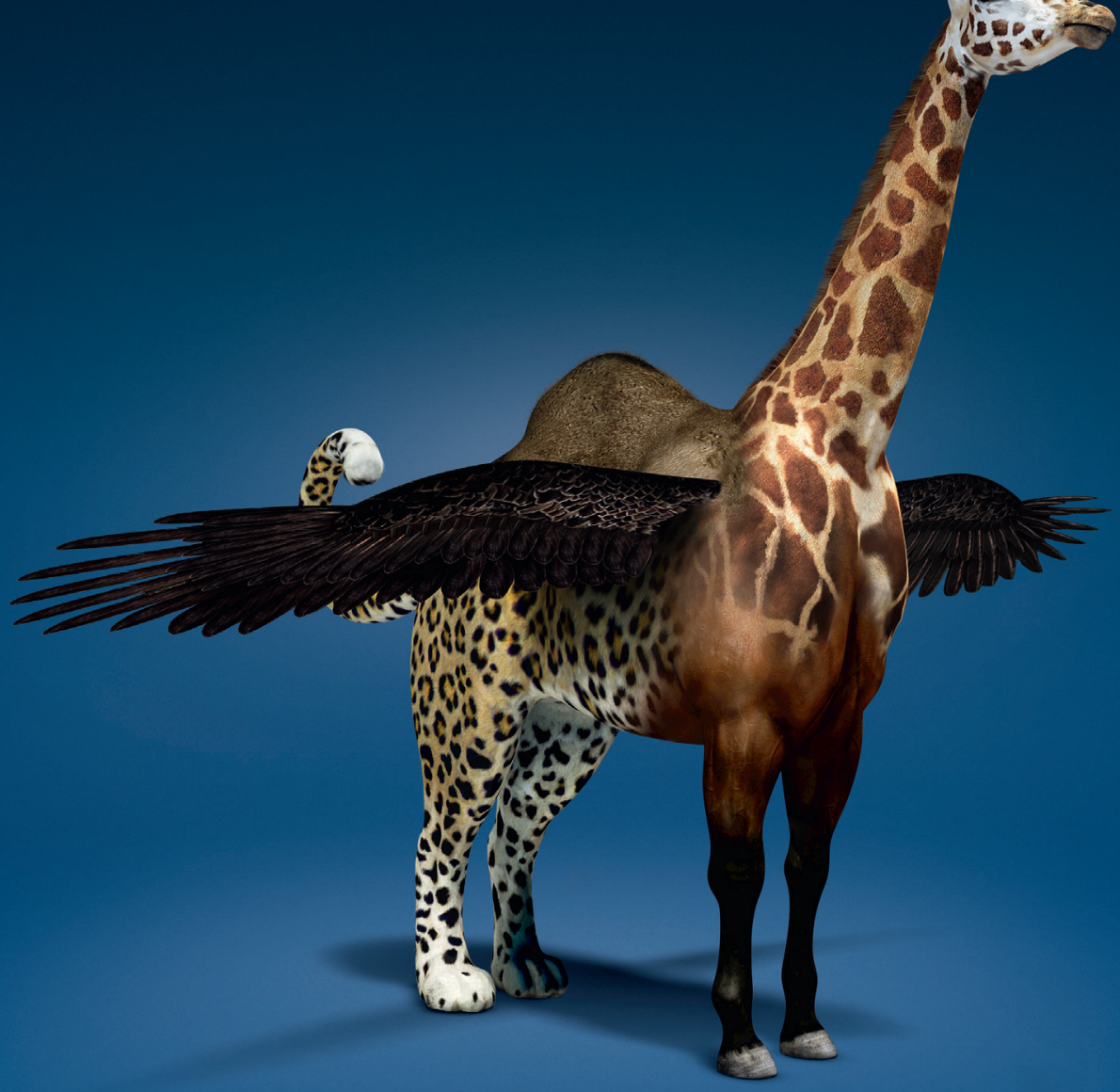

\section{5-on-1 reality: Bruker's range of analytical tools for SEM.}

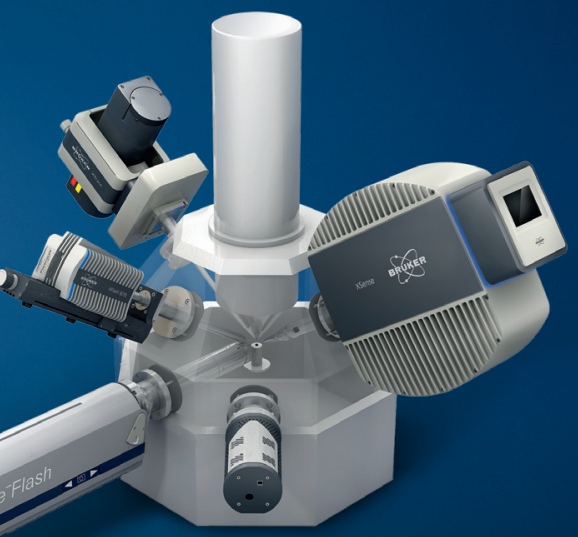

EDS, EBSD, WDS, Micro-XRF and Micro-CT - Bruker is the world's only manufacturer to offer five analysis methods for SEM. Plus, our new ESPRIT 2.0 software not only controls our QUANTAX EDS and QUANTAX CrystAlign EBSD but also, via its functional interface, our innovative new XSense WD spectrometer and XTrace micro-spot X-ray source. And because we know what you expect of us, we are already thinking about our next innovation. Someone has to be first.

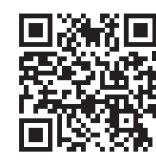

www.bruker-5on1.com

Innovation with Integrity 
Table 1: Responding biological microscopy centers.

\begin{tabular}{|c|c|c|c|c|c|c|c|}
\hline & US-East & US-Central & US-West & Canada & Europe & Asia-Pacific & Total \\
\hline \# Survey Responders & 48 & 33 & 19 & 10 & 51 & 14 & 175 \\
\hline 1 Microscopy Core & $37.5 \%$ & $41.9 \%$ & $44.4 \%$ & $22.2 \%$ & $60.0 \%$ & $50.0 \%$ & $42.7 \%$ \\
\hline 2 Microscopy Cores & 31.3 & 35.5 & 27.8 & 33.3 & 24.0 & 21.4 & 28.9 \\
\hline 3 Microscopy Cores & 10.4 & 16.1 & 5.6 & 11.1 & 10.0 & 21.4 & 12.4 \\
\hline 4+ Microscopy Cores & 20.8 & 6.5 & 22.2 & 33.3 & 6.0 & 7.1 & 16.0 \\
\hline 2 Systems or Less & 6.3 & 15.2 & 10.5 & 50.0 & 4.0 & 7.1 & 10.3 \\
\hline 3-5 Systems & 29.2 & 33.3 & 47.4 & 40.0 & 24.0 & 28.6 & 31.0 \\
\hline 6-10 Systems & 39.6 & 30.3 & 21.1 & 0.0 & 30.0 & 21.4 & 29.3 \\
\hline 10-20 Systems & 25.0 & 12.1 & 21.1 & 10.0 & 32.0 & 28.6 & 23.6 \\
\hline >20 Systems & 0.0 & 9.1 & 0.0 & 0.0 & 10.0 & 14.3 & 5.7 \\
\hline Wide-Field & 54.2 & 63.6 & 73.7 & 60.0 & 72.5 & 42.9 & 62.9 \\
\hline Confocal & 87.5 & 90.9 & 89.5 & 80.0 & 98.0 & 71.4 & 89.7 \\
\hline Wide-Field + Confocal & 52.1 & 60.6 & 68.4 & 50.0 & 72.5 & 42.9 & 60.6 \\
\hline Multi-photon & 52.1 & 45.5 & 31.6 & 30.0 & 52.9 & 50.0 & 47.4 \\
\hline SEM & 39.6 & 36.4 & 21.1 & 0.0 & 19.6 & 50.0 & 29.7 \\
\hline TEM & 41.7 & 45.5 & 21.1 & 10.0 & 23.5 & 57.1 & 34.3 \\
\hline EM + Light Microscopy & 33.3 & 33.3 & 21.4 & 0.0 & 21.6 & 35.7 & 26.7 \\
\hline Own Research YES & 58.3 & 53.1 & 31.6 & 60.0 & 45.1 & 28.6 & 48.3 \\
\hline
\end{tabular}

Note: The first section lists the percentage of institutions having 1 to $4+$ microscopy cores. The second section shows the percentage of cores that have certain types of instruments. The third section shows the percent of institutions that have various total numbers of systems. The last section indicates whether the cores conduct their own research.

Operating costs. Operating costs of cores vary a great deal and are clearly a reflection of the number of cores per institution, number and types of instruments and staff numbers, and, presumably, how different expense categories are accounted for in the financial systems of different institutions. Of all the possible correlations between annual operating costs and number of cores, type of instrument, and level of usage, the most appropriate was the number of instruments, even though the exact number of instruments by type were not solicited in the survey. Those with 1-2 instruments report a range of annual operating expenses of US\$ 50,000-80,000, those with
3-5 instruments US $\$ 120,000-220,000$, and with 6-10 instruments US $\$ 230,000-270,000$ per year. This yields an average of $\$ 40-50,000 /$ instrument/per year. Institutions with $>10$ instruments show substantial differences, mainly depending on the number of cores and the mix of instrument types.

Sources of operating funds. Survey participants were asked what percentage of funds originated from user fees, institutional support, grants, or other sources (Table 2). For most regions (except Canada) about one-third of a facility's operating expenses are covered by fees; less than 10\% report that all of their operating expenses are covered by fees

Table 2: Funding sources for core facility operational expenses.

\begin{tabular}{|l|c|c|c|c|c|c|c|}
\hline & Resp & Fees & Institution & Grants & Other & \multicolumn{2}{|c|}{$\begin{array}{c}\text { Institutional Funding is More } \\
\text { than Half of the Total }\end{array}$} \\
\hline & $\#$ & $\%$ & $\%$ & $\%$ & $\%$ & & $\%$ \\
\hline US-East & 45 & 30.0 & 46.6 & 16.6 & 6.8 & 26 & 57.8 \\
\hline US-Central & 33 & 38.6 & 46.0 & 13.4 & 2.0 & 13 & 39.4 \\
\hline US-West & 18 & 29.5 & 27.0 & 40.7 & 2.8 & 6 & 33.3 \\
\hline Canada & 10 & 19.0 & 35.5 & 30.5 & 15.0 & 3 & 30.0 \\
\hline Europe & 49 & 33.0 & 48.7 & 16.4 & 1.9 & 26 & 53.1 \\
\hline Asia-Pacific & 14 & 35.0 & 40.4 & 18.3 & 6.3 & 5 & 35.7 \\
\hline
\end{tabular}

Note: The second column denotes the number of responders to this question and may be compared to the total participants by region (Table 1). 
Table 3: Funding sources for new equipment.

\begin{tabular}{|l|c|c|c|c|c|c|c|c|}
\hline & Resp & G-\% & GI-\% & GF-\% & GIF-\% & I-\% & IF-\% & F-\% \\
\hline Asia-Pacific & 12 & 16.7 & 25 & 0 & 0 & 33.3 & 16.7 & 8.3 \\
\hline Canada & 9 & 66.7 & 0 & 11 & 0 & 0 & 0 \\
\hline Europe & 46 & 8.7 & 8.7 & 11 & 2.17 & 19.6 & 28.3 & 22 \\
\hline USA-Central & 33 & 12.1 & 39.4 & 3 & 15.2 & 15.2 & 9.09 & 6.1 \\
\hline USA-East & 44 & 13.6 & 50 & 0 & 18.2 & 11.4 & 2.27 & 4.5 \\
\hline USA-West & 19 & 26.3 & 31.6 & 11 & 5.26 & 5.26 & 15.8 & 5.3 \\
\hline
\end{tabular}

Note: These data show the percent of cores that receive funds from government sources only (G), government + internal institutional sources (Gl), government + foundations (GF), government + internal + foundations (GIF), internal institutional only (I), internal institutional + foundation (IF), and foundation money only (F).

(data not shown). Institutional support ranged from $27 \%-49 \%$ of operating expenses. For nearly half of the respondents, their institution funded more than half of the operating expenses. Financial resources from grant money for operational expenses seem to play a bigger role in US-West and Canada.

Funding sources for new equipment. A similar question concerned the sources of funds for capital equipment (Table 3). In most cases, cores obtained funding from three sources: government, internal institutional funds, and foundations; but, there are considerable differences between regions. In the US and Canada, government funding plays a large role, whereas in Europe and Asia Pacific internal sources and foundations contribute the largest percentage.

Microscope user fees. There are two categories of fees charged: for unsupervised/regular use of instruments and for supervised/training sessions. Some institutions charge no fees for use of microscopes. Tables $4 \mathrm{a}$ and $4 \mathrm{~b}$ present a detailed picture. Keep in mind that the number of responses has to be correlated to the number of cores that claim to have a particular instrument and not to the total number of responders (see Table 1). It is interesting to note that a large proportion of cores do not charge at all or charge hourly fees at the lower end of the spectrum (US\$10-30/hour), which does not easily correlate with the perceived challenges for the "business" of core microscopy - a question we will address later in this article.

Disposal of old instruments. Nearly all institutions responded to this question (individual data are not shown in a table). Depending on the instrument, directors make the following choices: about $30 \%$ do nothing, $40 \%$ trade in for a replacement system, $40 \%$ donate the instrument to other researchers, and about $25 \%$ sell to third parties. This question is clearly a businessrelated issue. The authors suggest that trade-ins, auctions, or the like could be places to get some return for an unused asset.

\section{Users of the Microscopy Cores}

Other than those cores doing their own research, the full attention of managers and staff is centered on support of users in their many different research goals, from the life sciences to engineering specialties. This section explores the number of individual Principal Investigator (PI) laboratories that the cores support, their primary areas of research, their weekly usage, and what kind of advanced microscopy techniques are being used.

How many labs use each instrument?. Without knowing the number of lab members from each lab that would use a particular instrument of a core, we could only correlate the number of PIs reported to use a core with the number of instruments in that core. The data (not shown in a table) exhibit a high level of consistency between the 3 US regions at 5.6 labs per instrument, for example, a core with 3-5 instruments would have 17-28 labs using the facility. Applying the same criteria to Canada, 2 labs per instrument were reported, Europe an average of 3.7 labs, and Asia Pacific 3.4 labs. The actual frequency of usage by PIs is not known, and the weekly instrument usage (next point) is difficult to relate to the present question.

Although the majority of centers do not have any industrial clients, a small number report up to $10 \%$ of their users come from outside the academic setting. Perhaps this is a potential source of income for microscopy cores, while making available their expertise to advance the goals of business enterprises.

Weekly usage by instrument. Table 5 details the usage results by instrument and by hours per week. We have refrained from over-interpreting the data by applying factors such as reduced numbers of days in a year (for example, public holidays/ semester calendars), time for maintenance/repair, and other reasons reducing utilization. Nevertheless, looking at the raw data and a realistic potential of 30 hours per week for an instrument's availability, there appears to be room for higher utilization rates. Taking the last two categories together (20-60 hours/week), only confocal microscopy-the "workhorse" of any center-is close to reaching potential by some $75 \%$ of centers. So, the questions that arise for all stakeholders (funding agencies, the universities, their core centers, and users) are the following: (1) Is the apparent underutilization of capacities a natural phenomenon of providing tools for researchers to advance their goals, irrespective of how many benefit from these tools? (2) Are researchers perhaps not aware of the potential of advanced microscopy techniques that could be beneficial to their work? (3) Are there barriers to the greater utilization of these instruments (for example, user fees, proximity)? (4) Are the rapid advances in technology reducing the lifespan of instruments, with some microscopes being superseded by even more sophisticated versions?

User's primary area of research. The details here (no table) hold no surprises, with $60-80 \%$ of respondents engaged in the life sciences (clinical research for 7-19\% of respondents); whereas, materials science uses varied by region: 3\% in Europe, $8 \%$ in Canada, $19 \%$ in Asia Pacific, and 15-23\% in the three US regions. 


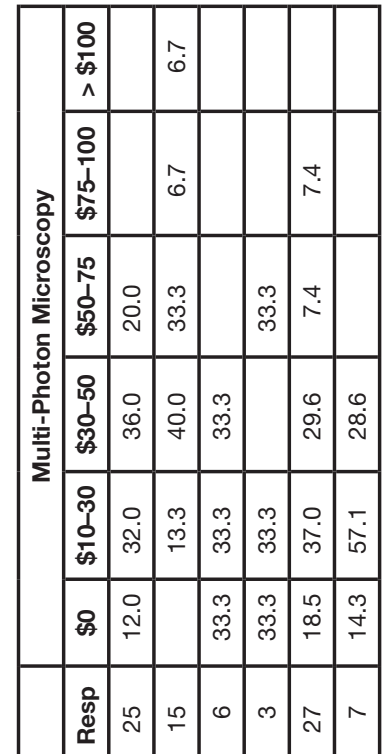

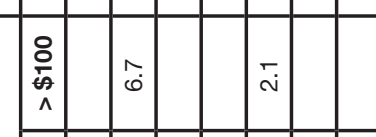

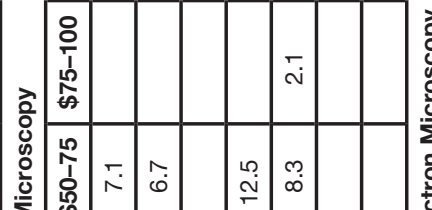

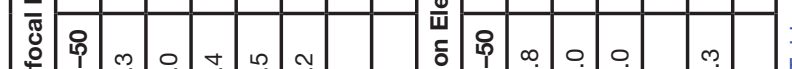

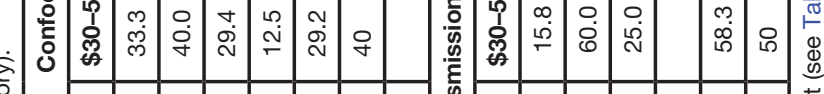

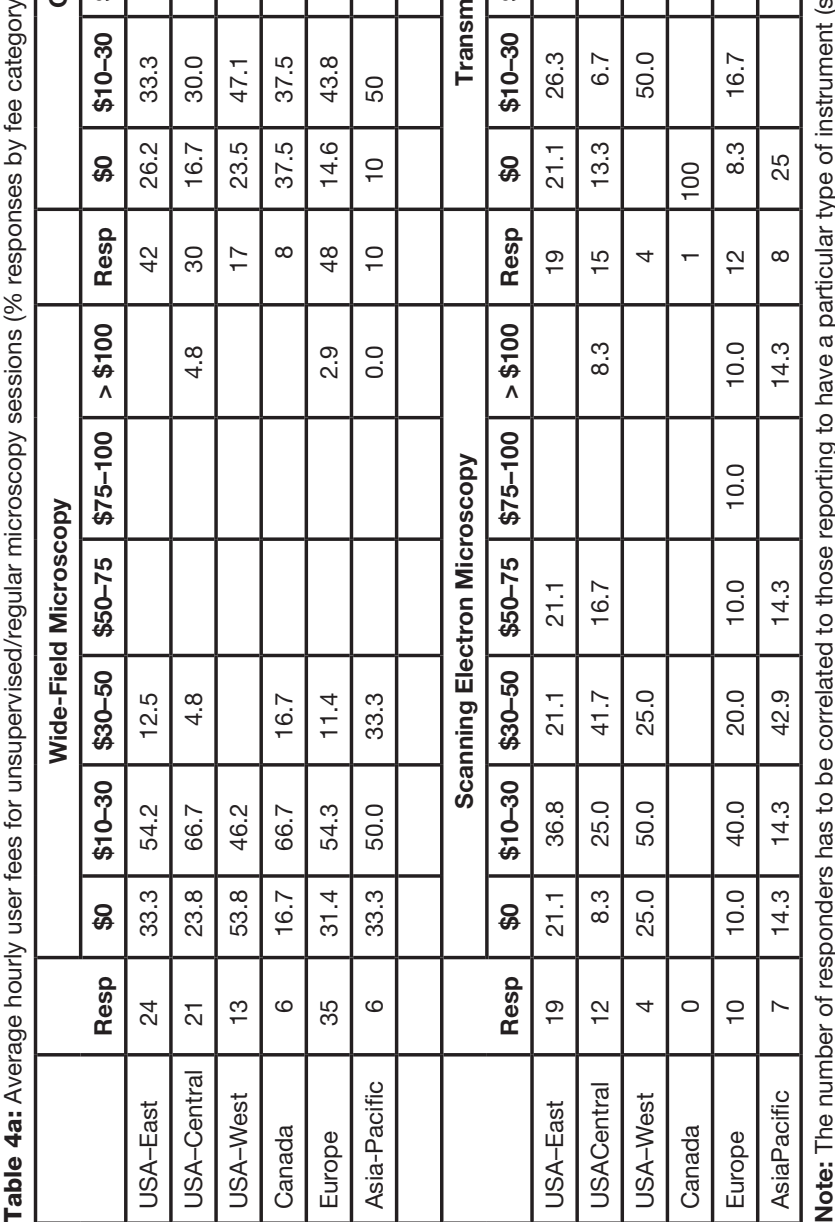

40
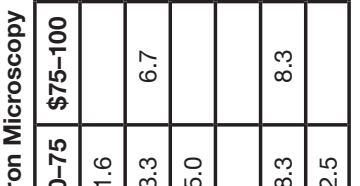

ì

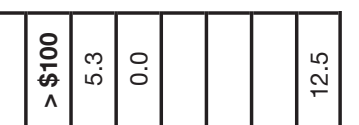

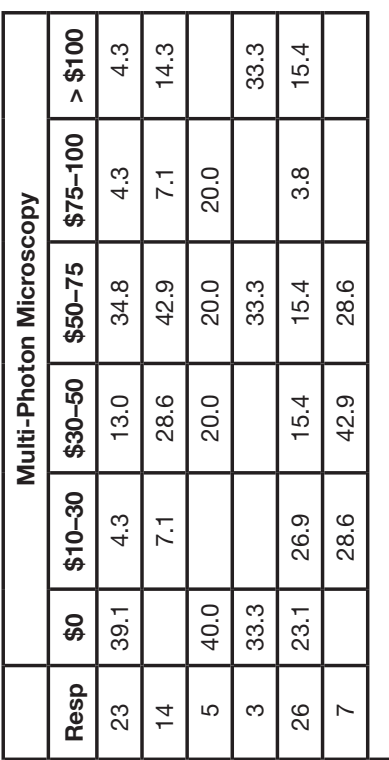

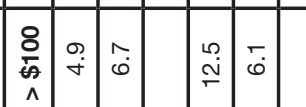

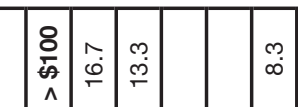

\begin{tabular}{|c|c|c|c|}
\hline 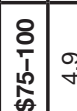 & 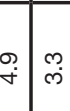 & ف & $\begin{array}{l}\text { ỹ } \\
\stackrel{0}{ }\end{array}$ \\
\hline
\end{tabular}

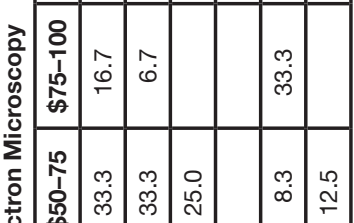

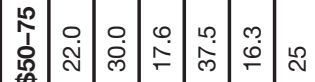

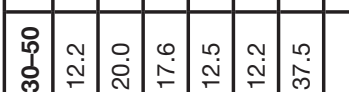

实

再

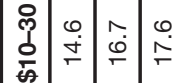

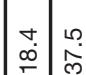

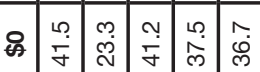

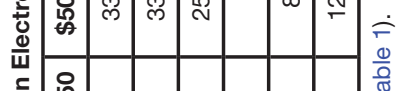

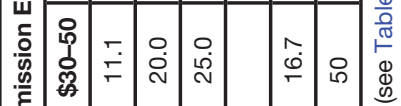

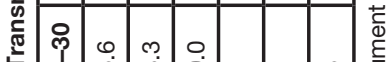

产

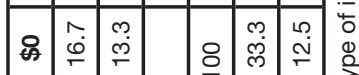

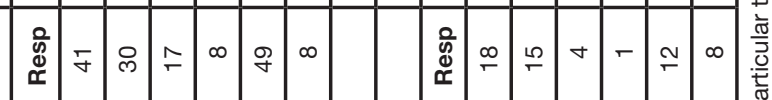

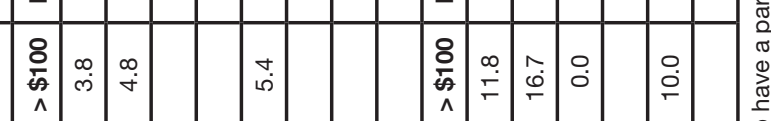

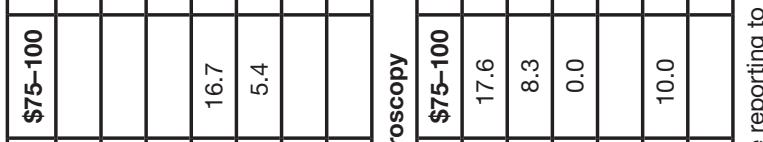

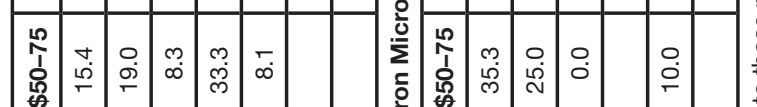

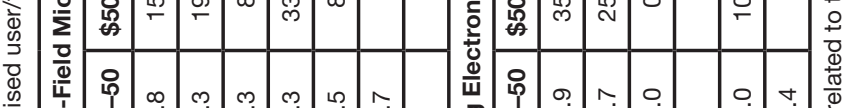

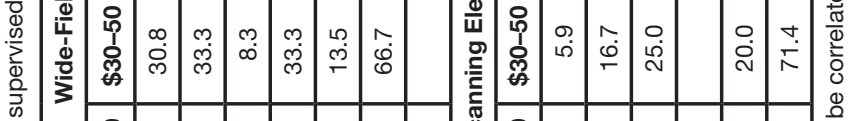

\begin{tabular}{|c|c|c|c|c|c|c|c|c|c|c|c|c|c|}
\hline & -1 & & & & $\stackrel{\sim}{\sim}$ & \ulcorner & $\frac{0}{6}$ & & $\stackrel{0}{\circ}$ & \multicolumn{4}{|l|}{ 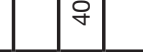 } \\
\hline 呙 & $\begin{array}{l}0 \\
\dot{m} \\
\dot{m}\end{array}$ & $\begin{array}{l}0 \\
\stackrel{0}{0} \\
\stackrel{\infty}{\sim}\end{array}$ & $\begin{array}{c}\infty \\
\infty \\
\infty \\
\infty \\
\infty\end{array}$ & $\begin{array}{l}\hat{\dot{\theta}} \\
\stackrel{2}{2}\end{array}$ & 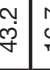 & $\begin{array}{l}\hat{\varphi} \\
\hat{\varphi}\end{array}$ & 8 & 亗 & $\hat{\leftrightarrow}$ & 怘 & & ?: & 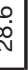 \\
\hline 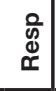 & $\stackrel{\oplus}{\sim}$ & $\bar{\sim}$ & $\cong$ & $0 ?$ & $\hat{m}$ & 0 & 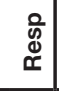 & 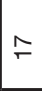 & $\stackrel{\simeq}{\sim}$ & $\nabla$ & 0 & 이 & 4 \\
\hline & 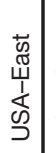 & 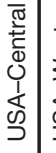 & 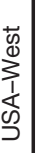 & $\begin{array}{l} \\
\frac{\pi}{0} \\
\mathbb{0} \\
\mathbb{0} \\
0\end{array}$ & & 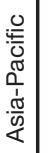 & & 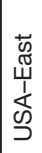 & 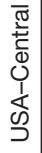 & 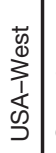 & $\begin{array}{l}\frac{\pi}{0} \\
\frac{\pi}{\pi} \\
0 \\
0\end{array}$ & | & \\
\hline
\end{tabular}

www.microscopy-today.com • 2014 March 


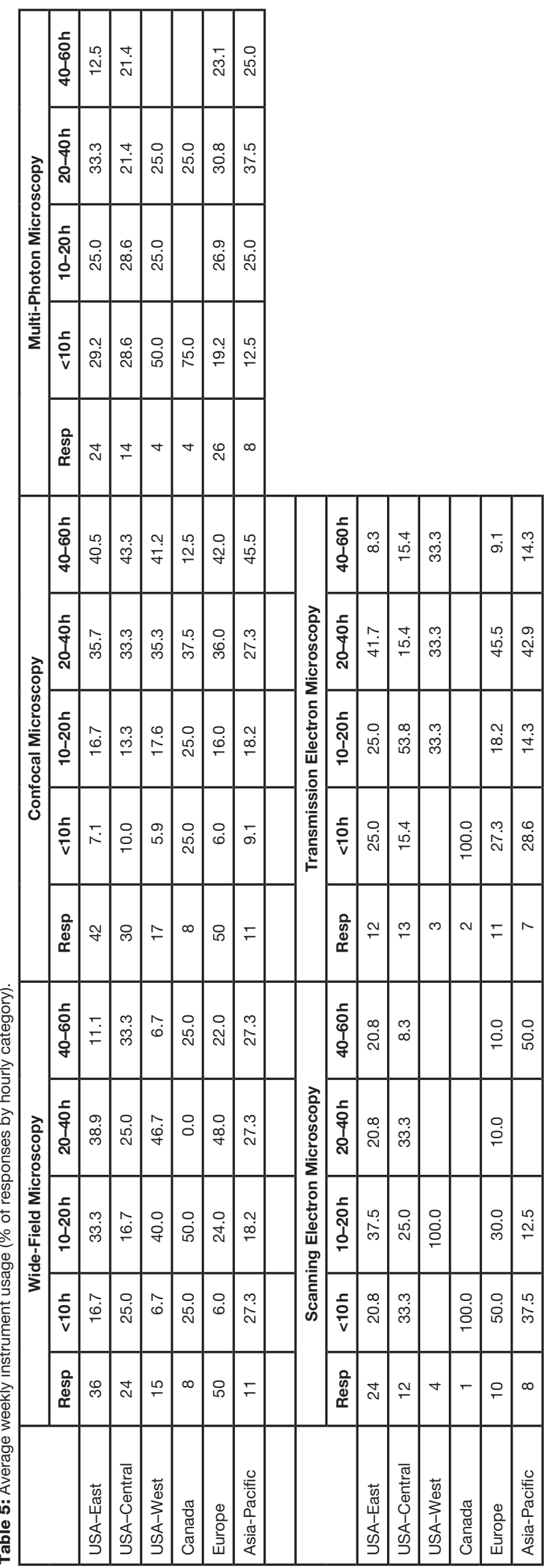

Advanced imaging techniques employed by users. Table 6 shows mainstream as well as specialized applications, confirming the wide-ranging capabilities of microscopy cores. The data do not contain the frequency of these applications, although a recent multi-year literature review shows an exponential increase in fluorescence resonance energy transfer (FRET) publications. (www.kcci.virginia.edu).

Based on the rate of innovation over the last decade in optics, lasers, detectors, ancillary hardware improvements, fluorophores and the like, it is safe to assume that each of the light microscopy categories will benefit from future technical advances. Super-resolution instruments and light sheet systems (also know as selective plane illumination microscopy [SPIM]), although occupying a specialist niche now, will probably find expanded applications.

\section{Management and Organizational Matters of Microscopy Cores}

As mentioned earlier, microscopy cores are a hybrid of academic endeavor and business excellence that serve sophisticated customers, provide competitive advanced systems, obtain funds for capital expenditures, and somehow balance operating expenses with income from various sources. Whatever the "business"-related challenges, even for those cores having 100\% financial support from some source (their institution, grants, etc.), the use of all modern tools available to run an efficient organization must be a priority. These include effective marketing to attract new users, having a firm handle on instrument utilization, a sound financial control system with straightforward feedback on "How well are we doing?", and savvy networking within the establishment to promote the value of the microscopy core.

What are the key challenges for microscopy core directors? The survey presented four options for this question: (1) "Instrumentation issues" (instrument service/repair issues/ costs), (2) "Cost Recovery," (3) "Day-to-Day Operational Challenges," (4) and "Getting more users." Table 7 highlights the commonalities and differences between regions and perhaps gives a glimpse of the actual concerns of core directors. "Instrumentation issues" (point 1 above) appears to be the number one challenge, which is not surprising because systems have to be maintained, problems solved, etc. The number two challenge is "Cost Recovery" in the US-less so elsewhere-with similar percentages as "Instrument Issues." Number three was "Day-to-Day Operations," and number four was "More Users." In dealing with the first three challenges, it would appear that marketing, tracking financials, and consulting should be addressed.

A surprising contradiction lies in the fact that "Cost Recovery" is not strongly linked to the level of priority for the challenge of "Getting more Users." Is "Getting more Users" an underdeveloped area in microscopy centers? Are core directors mainly concerned with marketing to users or are they looking for sources of cost recovery outside the fees they can charge existing users? Could they be expressing the feeling that fees are unlikely to ever cover operating costs, unless they become prohibitively high? "Day-to-Day Operational Challenges" 
Table 6: Advanced technologies used by microscopy centers in various regions (\% responses by technique).

\begin{tabular}{|l|c|c|c|c|c|c|}
\hline & US-East & US-Central & US-West & Canada & Europe & Asia-Pacific \\
\hline Responders & 45 & 28 & 16 & 9 & 48 & 13 \\
\hline FRET & 62.2 & 57.1 & 56.3 & 66.7 & 77.1 & 61.5 \\
\hline FLIM & 22.2 & 17.9 & 12.5 & 11.1 & 37.5 & 46.2 \\
\hline FRAP & 60.0 & 67.9 & 50.0 & 66.7 & 85.4 & 61.5 \\
\hline TIRF & 44.4 & 35.7 & 31.3 & 33.3 & 50.0 & 46.2 \\
\hline Correlative Microscopy & 35.6 & 14.3 & 12.5 & 11.1 & 27.1 & 46.2 \\
\hline Photo Activation & 44.4 & 53.6 & 43.8 & 66.7 & 70.8 & 53.8 \\
\hline Super Resolution & 22.2 & 10.7 & 31.3 & 11.1 & 27.1 & 38.5 \\
\hline FCS & 15.6 & 3.6 & 6.3 & 11.1 & 22.9 & 53.8 \\
\hline Raman Spectroscopy & 2.2 & 17.9 & & & 2.1 & \\
\hline X-Ray Microanalysis & 26.7 & 21.4 & 12.5 & & 6.3 & 23.1 \\
\hline Cryo-techniques & 20.0 & 25.0 & 6.3 & 11.1 & 6.3 & 23.1 \\
\hline EM Tomography & 22.2 & 28.6 & 12.5 & 22.2 & 16.7 & 15.4 \\
\hline $\begin{array}{l}\text { Electron Energy Loss } \\
\text { Spectroscopy (EELS) }\end{array}$ & 15.6 & 7.1 & & & & \\
\hline Other & 8.9 & 17.9 & 12.5 & 11.1 & 18.8 & \\
\hline
\end{tabular}

Note: Total number by responders has to be correlated to all responders and type of instruments (see Table 1). FLIM, TIRF, techniques requiring special systems, and electron microscopy are in their own class of imaging technologies. FRET = Förster Resonance Energy Transfer; FLIM = Fluorescence Lifetime Imaging Microscopy; FRAP = Fluorescence Recovery After Photobleaching; TIRF = Total Internal Reflection Fluorescence [Microscopy]; FCS = Fluorescence Correlation Spectroscopy; and EM = Electron Microscopy.

covers a wide range of possibilities, which were left to the responder to interpret.

How are the services of core facilities marketed? Figure 1 shows that there are three equally important venues: websites, lectures/seminars, and emails. Considering the paper by Farber and Weiss [3], "Core Facilities: Maximizing the Return on Investment," one is left to wonder what barriers exist within institutions and what opportunities exist outside the traditional home customer base (for example, neighboring institutions, research parks, industrial clients).

Productivity tools. A practical day-to-day management issue concerns microscope scheduling, training, and consultancy time, as well as the billing for these services where fees are charged. There are a number of areas in running a microscopy core that could benefit from systems and software that automate tasks: billing, reserving instrument time, tracking instrument usage, tracking teaching and consultancy time, and tracking instrument maintenance time/cost. These are important business tools to have available for assessing the effectiveness of the operation, fixing problem areas, and convincing backers/ financial supporters that their money was wisely spent. The majority of institutions answered these questions. The survey divided the scheduling and billing answers into "Manual," "Home-Built Software," and "Commercial Systems/Software." "Manual" means a personal call, a sheet on the microscope facility door, etc. The definition of "home-Built Software" covered anything that was created internally allowing users to book without personal contact with the core (such as Google Calendar). "Commercial" refers to special-purpose software, usually integrating scheduling with billing (and providing statistics), such as that available from Idea Elan and other companies.
Table 8 displays the results for scheduling and billing. On the scheduling side, most cores (except Asia Pacific) have developed some homebuilt automated system or are using freely available software like Google Calendar. A surprisingly high percentage of responders still rely on some sort of manual system. Commercial software is highly favored in the Asia-Pacific region (70\%), compared to an average of about $30 \%$ in the rest of the world.

The billing side of Table 8 shows an equally varied picture. Clearly, the manual and home-built methods of billing are most widely used. This means that scheduling and billing are not integrated and require further processing, which leaves room for streamlining and increased efficiencies. Key benefits of commercial core facility management software are the ability to perform the most essential activities: scheduling, managing sample submissions, usage tracking, invoicing, integration with authentication and financial systems, multi-facility management, and managing different user roles along with extensive configuration options all under one web interface.

\section{Discussion}

This survey provides some valuable insights into the workings of microscopy core centers, while providing practical information for their directors and staff. As outlined and implied by other authors referenced in this paper, the nature of an imaging center requires a healthy dose of business savvy: considering questions of return on investment, resource utilization, acquiring more clients, and increasing existing users' frequency. "Instrument issues" and "cost recovery" were reported to be the top challenges for core directors, followed by "operational issues" and the need for "more users." All four 


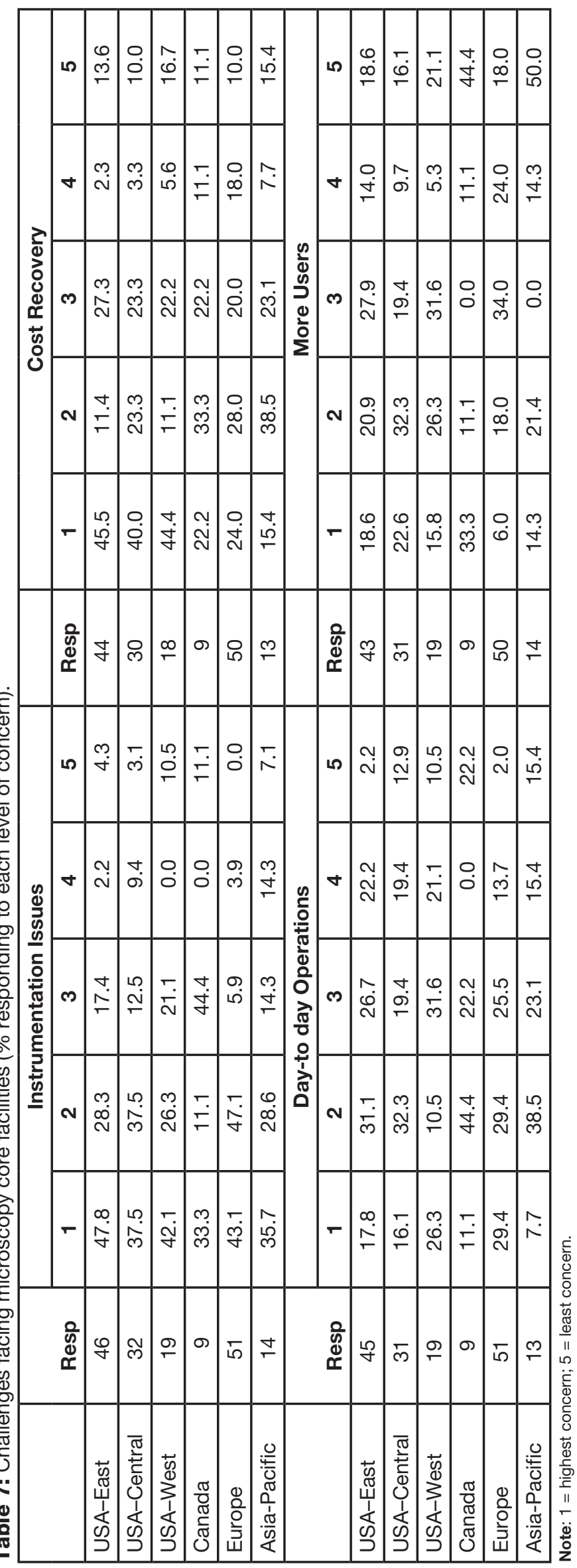

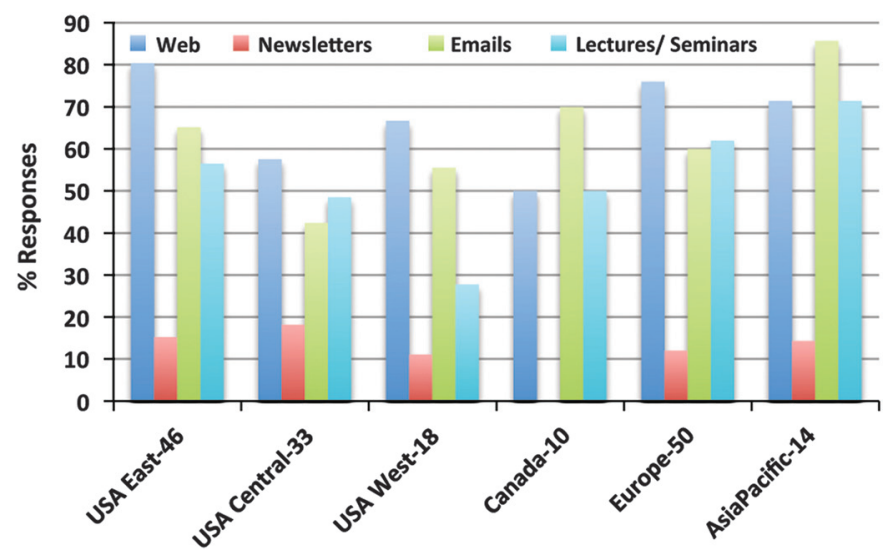

Figure 1: Marketing the core facility's services. Most regions use all four categories of communications: websites (blue), newsletters (red), emails (green) and lectures/seminars (teal). Numbers attached to regional labels denote the number of responders.

challenges are connected and should concern all stakeholders in the current economic environment, which is unlikely to get any easier for institutions, funding agencies, users, suppliers, and microscopy core directors. Institutions have opportunities to streamline and consolidate their imaging resources to avoid duplication. Funding agencies already apply criteria to avoid duplication, but this is difficult to execute. Users are also affected by funding issues and can help their institutions migrate to more economically structured core facilities. Suppliers also have a degree of self-interest in a healthy customer base: in spite of their bottom-line obligations and high development costs, they must have an interest in lowering customer costs. Finally, core directors are obliged to run an efficient and effective operation to achieve transparency of financial aspects, attract and retain satisfied users, and persuasively communicate with their institutions and funding agencies. For that, they need facts and information at their fingertips about all aspects of the operation. The survey results suggest that there is considerable room for improvement toward integrating the user-, instrument- and finance-related factors into one seamless information system.

\section{Conclusion}

Microscopy centers in institutional settings must achieve academic excellence and simultaneously operate as a successful business in terms of scheduling, training, and financial control. The results of this survey of 175 microscopy core facilities in 22 countries presents a snapshot of various practices dealing with these challenges. Core directors have an opportunity to compare their own center's performance with summary data for centers in the US, Canada, Europe, and the Asia-Pacific region. Instrument issues and cost recovery were ranked as the most important challenges for core directors, followed by daily operations and the need for more users. Scheduling of instrument time and billing of users are handled by home-built software, commercial software, and manual methods. Because there is still significant use of manual methods for billing, there is 
Table 8: Management of scheduling and billing at microscopy core facilities (\% responding to each category).

\begin{tabular}{|l|c|c|c|c|c|c|}
\hline & \multicolumn{3}{|c|}{ Scheduling } & \multicolumn{3}{c|}{ Billing } \\
\hline & Manual & Home & Commercial & Manual & Home & Commercial \\
\hline USA-East & 35.3 & 32.4 & 32.4 & 32.6 & 37.2 & 30.2 \\
\hline USA-Central & 20.0 & 48.0 & 32.0 & 28.6 & 46.4 & 25.0 \\
\hline USA-West & & 61.1 & 38.9 & 58.8 & 17.6 & 23.5 \\
\hline Canada & 33.3 & 66.7 & & 55.6 & 44.4 & \\
\hline Europe & 7.3 & 70.7 & 22.0 & 28.9 & 53.3 & 17.8 \\
\hline Asia-Pacific & 10.0 & 20.0 & 70.0 & 28.6 & 35.7 & 35.7 \\
\hline
\end{tabular}

Note: Scheduling and billing were defined as "manual" (a phone call to book, list on the door, a book to record usage), "home-built" (adaptation of free software, e.g. Google Calendar, spreadsheet/macro for billing), or "commercial" software integrating all activities (e.g., from Idea Elan and other companies).

considerable scope for improved efficiencies through the use of advanced commercial software. Moreover, instruments in many facilities are still not used to their complete potential. This provides an opportunity for both facility directors and researchers to further use the capital instruments to accelerate research.

\section{Acknowledgments}

We acknowledge Ms. Janani Kalyanakrishnan of Idea Elan and Dr. Krik Czymmek, former BioImaging Facility Director at the University of Delaware, for their help in preparing this manuscript.

\section{References}

[1] R Hale. A Framework for Managing Core Facilities within the Research Enterprise. Journal of Biomolecular Techniques 20 (2009) 226-30.

[2] S DeMaggio. "Running and setting up a confocal microscope core facility," in Methods in Cell Biology, vol. 70, Elsevier, New York 2002) 475-85, Chap. 14.

[3] GK Farber and L Weiss, Sci Transl Med 3(95) 2011, p. $95 \mathrm{~cm} 21$.

a powerful, multi-color, solid-state illuminator

why buy a lamp when you can have a light engine?

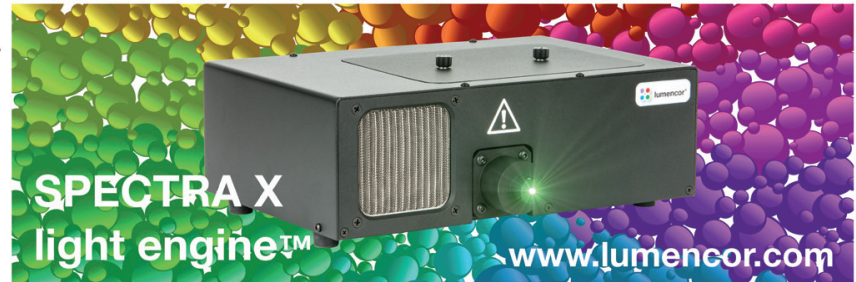

specific outputs are a function of instrument parameters results will vary

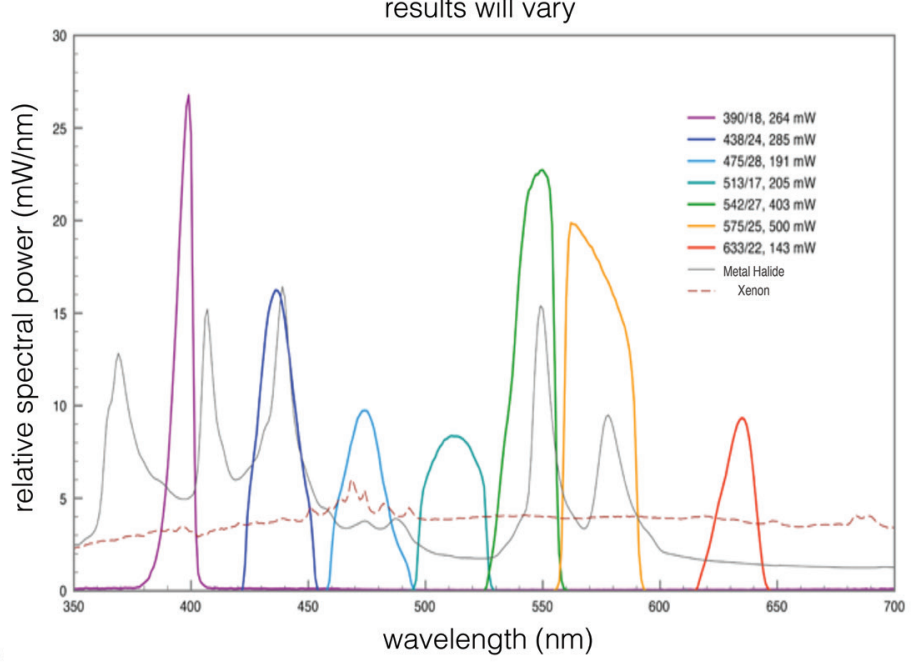


